\title{
Application of dye-ligands affinity adsorbent in capturing of rabbit immunoglobulin G
}

\begin{abstract}
The applicability of dye-ligands attached to an expanded bed chromatography quartz base matrix (Streamline ${ }^{\mathrm{TM}}$ ) for the affinity bioseparation of rabbit immunoglobulin $\mathrm{G}$ ( $\mathrm{IgG}$ ) was investigated. Reactive Green 5 (RG-5) immobilized onto adsorbent was selected for capturing of rabbit-IgG due to its higher binding capacity compared to other dye-ligands possessing similar ligand density. Adsorption parameters such as $\mathrm{pH}$, temperature, ionic strength and initial rabbit-IgG concentration were optimized for the adsorption of rabbit-IgG on the RG-5immobilized adsorbent. The highest rabbit-IgG adsorption was recorded in $\mathrm{pH} 7.0$, while the maximum binding capacity for BSA was achieved at $\mathrm{pH}$ 4.0. The adsorption of rabbit-IgG on RG-5-immobilized adsorbent was declined as the increase of ionic strength. There is no significant influence of temperature against adsorption efficiency of RG-5-immobilized adsorbent for rabbit-IgG. The adsorption phenomenon of rabbit-IgG on RG-5-immobilized adsorbent appeared to follow the Langmuir-Freundlich adsorption isotherm model. The theoretically maximum binding capacity (qm) of RG-5-immobilized adsorbent estimated from this isotherm was $49.3 \mathrm{mg} \mathrm{ml}-1$, which is very close to that obtained experimentally (49.0 mg ml-1). About 50\% of bound BSA on RG-5-immobilized adsorbent in binary adsorption system was removed with washing buffer containing $1 \mathrm{M} \mathrm{NaCl}$.
\end{abstract}

Keyword: Affinity; Bioseparation; Dye-ligand; Expanded bed chromatography; Immunoglobulin G purification; Adsorption 